\title{
Avatars rendering and its effect on perceived realism in Virtual Reality
}

\begin{abstract}
Immersive virtual environments have proven to be a plausible platform to be used by multiple disciplines to simulate different types of scenarios and situations at a low cost. When participants are immersed in a virtual environment experience presence, they are more likely to behave as if they were in the real world. Improving the level of realism should provide a more compelling scenario so that users will experience higher levels of presence, and thus be more likely to behave as if they were in the real world. This paper presents preliminary results of an experiment in which participants navigate through two versions of the same scenario with different levels of realism of both the environment and the avatars. Our current results, from a between subjects experiment, show that the reported levels of quality in the visualization are not significantly different, which means that other aspects of the virtual environment and/or avatars must be taken into account in order to improve the perceived level of realism.
\end{abstract}

Index Terms - avatars rendering, VR, perceived realism

\section{INTRODUCTION}

With the increasing popularity of virtual reality and the more affordable devices, many applications related to different disciplines have been developed in recent years. Immersive virtual reality solutions minimize the costs and risks associated with simulations in real environments. In this type of applications, the users wear a head mounted display and are fully immersed in a virtual world. They cannot see the real world nor their own body or the other potential participants. Virtual reality applications must face important challenges depending on the type of problem they want to solve. For instance, simulation of an emergency situation in VR needs to be as close as possible to reality, and the scenario should be crowded with avatars that look and behave as humans would do in real life.

To create plausible VR environments, several visualization techniques can be applied such as global illumination, dynamic shadows, ambient occlusion or physically based rendering materials. Avatars should also look as humanly as possible to enhance the plausibility of the virtual situation, which involves photo-realistic textures, high quality meshes and good rigging. Some of the most popular games can provide high levels of engagement by offering not only very challenging and entertaining goals, but also having highly realistic environments and avatars with responsive interaction to the player actions [1, 2]. However, achieving such levels of realism, has a very high cost, as it requires many months or even years of work from a large team of expert programmers and artists. It is thus, very difficult to provide those levels of realism for research experiments, where teams are smaller and typically lack having artistic experience (in modelling, illumination, rigging, animation).
Recently there has been some effort to provide photorealistic avatars with high quality textures, rigging and blend shapes [3] for the research community. It is thus possible at this stage, to evaluate to what extent those avatars can improve the reported levels of presence and realism of the virtual environment. For this purpose, we run an experiment with two scenarios depicting the same situation: an evacuation of a train station. One condition had low quality rendering, while the other offer high quality rendering. We hypothesized that the appearance of the avatars alone, should make a difference in the reported levels of perceived realism. However, we found no significant differences in the results, which implies that further analysis must be done to determine the reasons behind this findings, and what exactly is still missing.

\section{RELATED WORK}

There has been a large amount of work showing that having agents' reacting to the user by moving away and avoiding collisions, can enhance presence [4]. Similarly, if agents appear to interact by playing the correct animations when facing the user [5], or addressing the user by talking [6], can also greatly increase presence. The work by Bruneau et al. studied collision avoidance of a user immersed in a VR setup and reported differences in the user movement based on the appearance of the virtual characters [7].

Lighting, shape and materials play an important role in the transmission of human emotions. As constated by Wisessing et al. [8], combining brightness and changing the direction of the light affect the perception of the participants about the shape of the avatar's faces and their facial expressions. Shading can be used to express both positive and negative emotions: brighter conditions are related to happiness while darker lighting conditions are related to sadness. On the other hand, Zell et al. analyzed how shape and material could affect the perception of different facial expressions such as sadness, anger, happiness and surprise ([9]). They compared cartoonish, medium and high realistic characters. Their results showed that shape is the main factor for the perceived realism and that some expressions are perceived more intense with cartoon shapes.

Zibrek et al. [10] studied the effects that characters appearance had when playing video games. Their results showed that affinity towards virtual characters appears to be a complex interaction between the character's appearance and the exhibited personality. They also found that realism was in fact a positive choice for virtual characters in virtual reality, despite the typical problems involving the Uncanny Valley 

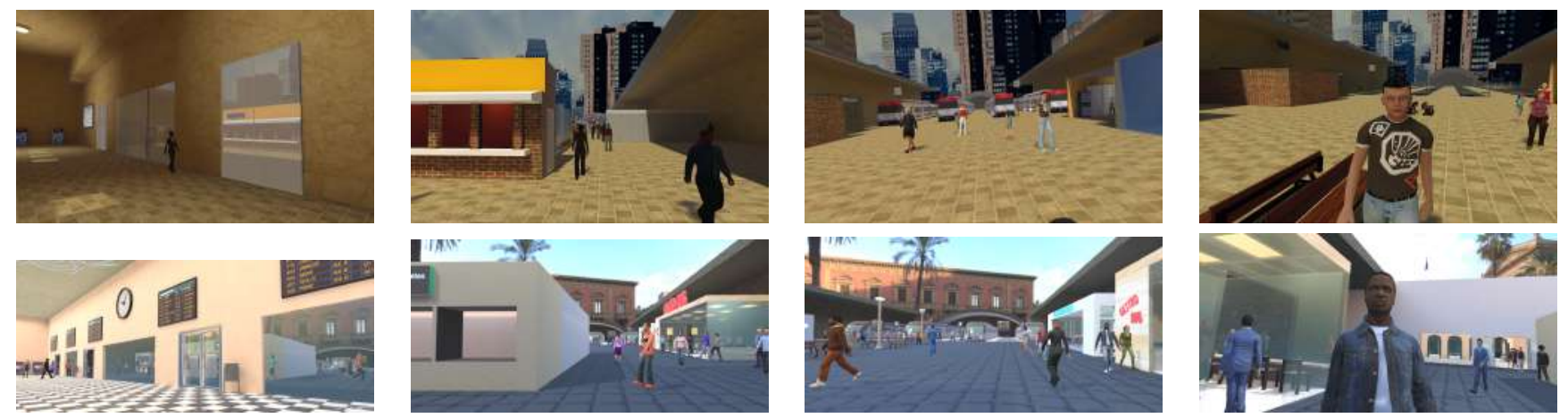

Fig. 1: Images showing at the top row the lower quality rendering (LQR) and at the bottom row the higher quality rendering (HQR) of the crowded train station

effect. Another study explored how photorealistic characters showing emotions would affect the responses of participants ([11]). They performed an experiment in which an avatar rendered with high, medium and low realism interacted with the participants in several situations: showing a sad, friendly and unfriendly attitude. They found that, for some emotions, the participants felt more concern for the high realism style compared to the other two.

In another study Zibrek at al. found that photorealism increases the self-reported measures of social presence and place illusion in VR, while no effect on the behavioural measure of proximity was found [12]. However, they noticed that the generalization of those findings applied to characters of limited interaction with the user, such as those in immersive movies, while we are interested in being able to interact with them.

\section{EXPERIMENT}

The goal of our pilot experiment is to evaluate to what extent the reported levels of presence and realism are increased by using higher quality rendering of avatars against lower quality ones. Two versions of the same virtual environment from the work by Rios et al. (2020) [13] have been used to run the experiment. The first version is a lower quality rendering (LQR) train station crowded with about one hundred lower quality rendering looking avatars (see Figure 4), while the second one is an improved version of the same train station with one hundred avatars with higher quality rendering (HQR) from the Rocketbox library [3] (see Figure 3). In order to match their appearance, the station has been enhanced with global illumination, an improved environment map, higher quality textures, and physically based rendering materials (see figure 1 for a visual comparison). Even though we wanted to study exclusively the impact of the avatars rendering, we increased the realism of both the environment and the avatars, to avoid the awkward effect of having higher quality rendering avatars in a lower quality rendering environment. Nevertheless, dynamic shadows have been kept in both scenarios since this has already been observed to have an impact on the reported levels of presence as shown in Slater (2016) [14], so we did not want our results differences to be based on that feature.

\section{A. Procedure}

Before executing the experiment, participants signed a consent form and filled out a form with demographic data including their experience with virtual reality and videogames. They also were warned about the possibility of dizziness while performing navigation using an HMD, and that they could stop the experiment if needed at any time. Then they were instructed on how to use the controller to interact with the virtual objects and to navigate through the environment.

When the simulation starts the user is located on the street in front of the main entrance of the train station. In both scenarios, participants were asked to perform several tasks that are shown to the participant in a semitransparent text box overlapping the virtual environment for a few seconds, and located at the user eye level. Once a task is performed, the next task is shown (see Fig. 2). Performing such sequence of tasks, forces the participant to move around the train station and meet the virtual avatars. When the last task is performed, a fire breaks out in one of the trains, an alarm goes off while a red light starts flashing and all the avatars start running towards one of the exits.

After the experiment, all the participants filled a questionnaire with 3 questions ranked in a Likert scale from 0 to 9. The questions can be found in table I.

TABLE I: Questionnaire

\begin{tabular}{|l|l|}
\hline Q\# & Question/Statement \\
\hline Q1 & The overall quality of the visualization was good. \\
\hline Q2 & The virtual humans' movement and appearance looked realistic. \\
\hline Q3 & $\begin{array}{l}\text { The behavior of the other avatars when the alarm went off made } \\
\text { me feel nervous. }\end{array}$ \\
\hline
\end{tabular}

\section{B. Physical Set-up}

All the experiments were conducted in a $1.5 \times 2 \mathrm{~m}$ indoor lab area since it is not necessary for the participants to move physically. Position tracking was performed using an HTC VIVE immersive virtual display and a VIVE controller was used to navigate the virtual space and to carry out the different 
tasks. The surroundings can be inspected by moving the head. The participants also wore earphones to hear the typical sounds of a train station (train announcements, people talking, steps, etc.). Unity Game Engine was used to render the environment and animate the avatars.

\section{Participants}

We performed a between subjects experiment with two groups of 13 participants in the LQR scenario and 13 in the HQR scenario. A summary of their characteristics can be found on table II.

TABLE II: Participants' characteristics

\begin{tabular}{|c|c|c|c|c|c|}
\hline & \%Male & \%Female & Min age & Max age & Avg \\
\hline LQR & 84.6 & 15.4 & 18 & 70 & 28.5 \\
\hline HQR & 92.3 & 7.7 & 20 & 34 & 22.8 \\
\hline
\end{tabular}

In both groups 11 participants ranked their level of experience in videogames between 6 and 9 , so we consider them as gamers.

\section{RESUlts}

In this section we describe the results obtained from our study. Each participant tried the experiment only once in one of the two possible scenarios. We analyzed the values gathered from the questionnaires and created the boxplots shown in figure 5. The means for each question can be found in table III .

TABLE III: Means for each question

\begin{tabular}{|c|c|c|}
\hline & LQR & HQR \\
\hline Q1 & 7.15 & 6.69 \\
\hline Q2 & 6.53 & 5.92 \\
\hline Q3 & 4.92 & 3.5 \\
\hline
\end{tabular}

As can be observed, all the means are lower for the $H Q R$ experiment than for the LQR experiment. A further study should be performed with more participants performing the experiments to check if there exists a statistically significant median between the LQR and the HQR groups (a Wilcoxon

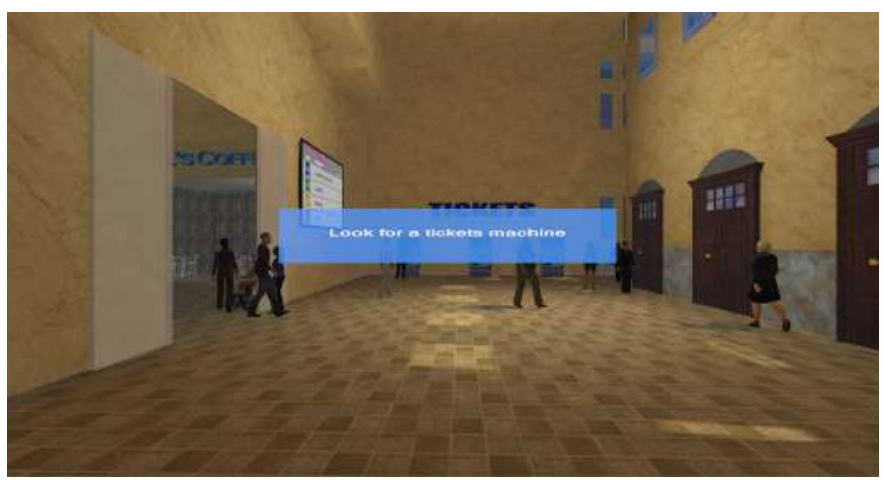

Fig. 2: Example of how the next task to perform is shown to the user.
Signed Rank Test should be performed, but it needs at least 20 participants in each group to get reliable results).

At first, our results seem surprising, when compared to some previous studies in the literature, such as those by Zibrek et al. [12]. However, most previous studies have been performed as within subject, where participants are exposed to all the different avatars' rendering. On the contrary, our study was performed as between subjects, and thus participants did not have a base line to compare against. This could explain the observed results. It is possible that lower quality rendering characters were perceived as having high quality for such a simple rendering, whereas participants had higher expectations regarding realism for photorealistic avatars and thus scored them with lower values.

\section{CONCLUSIONS AND FUTURE WORK}

Our preliminary study has shown that realistic rendering of the environment and the avatars does not appear to increase the perception of realism in the participants which is consistent with the work presented by Slater et al. [16]. This pilot experiment has been performed mostly with gamers, which are used to higher quality rendering game environments. Therefore, we are concerned that this could have an impact on their perceived realism, which lead to lower scores than we predicted. In spite of this, it is interesting to observe that it seems there is not an important difference in the realism scores between the experimental conditions. As a matter of fact, the realism perceived on the second condition was slightly lower for the environment, although similar for the avatars. Since it is difficult to separate appearance from behavior, we believe that it is very likely that further improving the avatars' behavioral response will also increase the reported levels of perceived realism and presence, as it will be less likely to suffer from the Uncanny Valley effect. We want to further continue this work, by performing a larger experiment including more questions, but also the evaluation of behavioral responses from the participants, such as for example, personal distance.

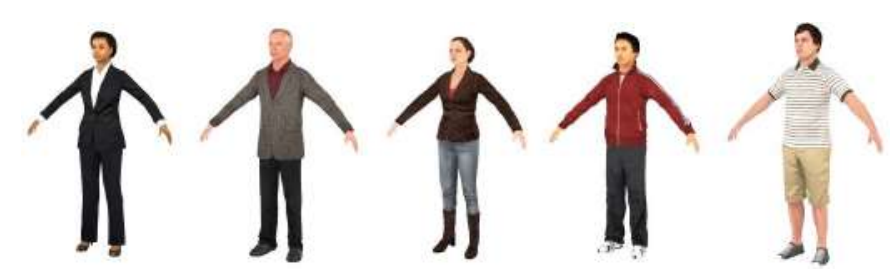

Fig. 3: A few example avatars from the Rocketbox library [3]

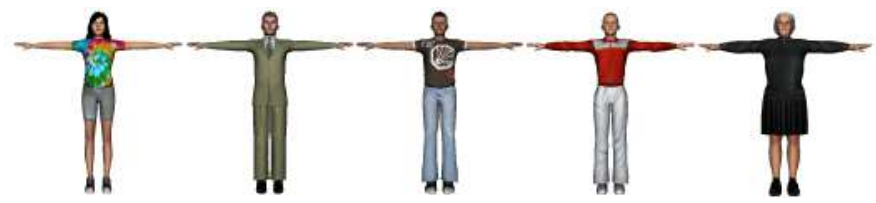

Fig. 4: A few example avatars from the Adobe Character Generator Tool [15] 

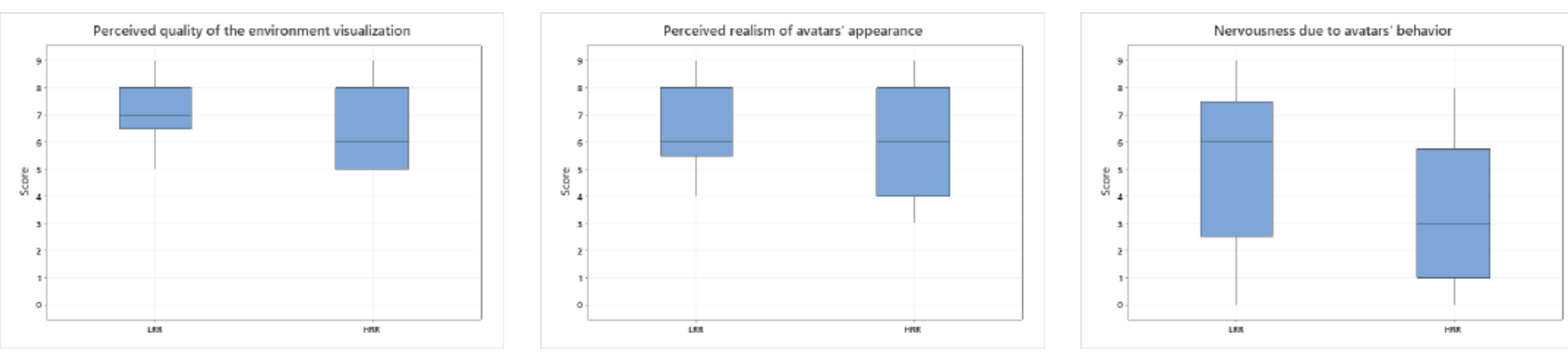

Fig. 5: Boxplots from the values gathered through the questionnaires

Since it is well known that interactive responses from the avatars or even having dynamic shadows, can have an impact on the responses of participants, we conclude that other aspects like better avatar animations would affect the perceived realism and nervousness of the participants. Therefore, we believe that higher quality rendering avatars will improve presence as long as other aspects that require movement and behavior are also improved.

\section{REFERENCES}

[1] Uncharted 4. Naughty Dog, 2016.

[2] The last of us Part II. Naughty Dog, 2020.

[3] M. Gonzalez-Franco et al. "The Rocketbox library and the utility of freely available rigged avatars for procedural animation of virtual humans and embodiment". In: Frontiers in Virtual Reality - Technologies for VR (to be published) (2020).

[4] Núria Pelechano Gómez et al. "Feeling crowded? exploring presence in virtual crowds". In: Proceedings of PRESENCE 2007. 2007, pp. 373-376.

[5] Marios Kyriakou, Xueni Pan, and Yiorgos Chrysanthou. "Interaction with virtual crowd in Immersive and semiImmersive Virtual Reality systems". In: Computer Animation and Virtual Worlds 28.5 (2017).

[6] M. Volonte et al. "Effects of Interacting with a Crowd of Emotional Virtual Humans on Users' Affective and Non-Verbal Behaviors". In: 2020 IEEE Conference on Virtual Reality and 3D User Interfaces (VR). 2020, pp. 293-302.

[7] Julien Bruneau, Anne-Helene Olivier, and Julien Pettre. "Going through, going around: A study on individual avoidance of groups". In: IEEE transactions on visualization and computer graphics 21.4 (2015), pp. 520528.

[8] Pisut Wisessing et al. "Enlighten Me: Importance of Brightness and Shadow for Character Emotion and Appeal". In: ACM Transactions on Graphics (TOG) 39.3 (2020), pp. 1-12.

[9] Eduard Zell et al. "To Stylize or Not to Stylize? The Effect of Shape and Material Stylization on the Perception of Computer-Generated Faces". In: ACM Trans. Graph. 34.6 (Oct. 2015). ISSN: 0730-0301. DOI: 10 .
1145/2816795.2818126. URL: https://doi.org/10.1145/ 2816795.2818126.

[10] K. Zibrek, E. Kokkinara, and R. Mcdonnell. "The Effect of Realistic Appearance of Virtual Characters in Immersive Environments - Does the Character's Personality Play a Role?" In: IEEE Transactions on Visualization and Computer Graphics 24.4 (Apr. 2018), pp. 1681-1690. ISSN: 1077-2626. DOI: 10.1109/TVCG. 2018.2794638 .

[11] Katja Zibrek, Sean Martin, and Rachel McDonnell. "Is Photorealism Important for Perception of Expressive Virtual Humans in Virtual Reality?" In: ACM Trans. Appl. Percept. 16.3 (Sept. 2019). ISSN: 1544-3558. DOI: 10 . 1145 / 3349609. URL: https : / / doi . org / 10 . 1145 / 3349609.

[12] Katja Zibrek and Rachel McDonnell. "Social presence and place illusion are affected by photorealism in embodied VR". In: Motion, Interaction and Games. 2019, pp. $1-7$.

[13] Alejandro Ríos and Nuria Pelechano. "Follower behavior under stress in immersive VR". In: Virtual Reality (2020), pp. 1-12.

[14] Mel Slater and Maria V Sanchez-Vives. "Enhancing our lives with immersive virtual reality". In: Frontiers in Robotics and AI 3 (2016), p. 74.

[15] AdobeCharacterGenerator. 2018. URL: https : / / charactergenerator.autodesk.com/.

[16] Mel Slater et al. "Visual realism enhances realistic response in an immersive virtual environment". In: IEEE computer graphics and applications 29.3 (2009), pp. 76-84. 